\title{
Structural Basis for Map Formation in the Thalamocortical Pathway of the Barrelless Mouse
}

\author{
Fulvia Gheorghita, Rudolf Kraftsik, Roger Dubois, and Egbert Welker \\ Département de Biologie Cellulaire et de Morphologie, Faculté de Médecine, Université de Lausanne, CH-1005 Lausanne, Switzerland
}

\begin{abstract}
Barrelless mice (BRL) homozygous for the BRL mutation that disrupts the gene coding for adenylyl cyclase type I on chromosome 11 lack spatial segregation of layer IV cortical cells and of the thalamocortical axons (TCAs) into barrel domains. Despite these morphological perturbations, a functional topographic map has been demonstrated. We reconstructed individual biocytin-injected TCAs from thalamus to barrel cortex in NOR (normal) and BRL mice to analyze to what extent the TCA arborization pattern and bouton distribution could explain the topographic representation of the whisker follicles. In BRL, the geometry of TCA is modified within layer IV as well as in infragranular layers. However, in both strains, the spatial distribution of TCA in layer IV reflects the spatial relationship of their cell bodies in the ventrobasal nucleus of the thalamus. The morphometric analysis revealed that TCAs of both strains have the same length, branch number, and number of axonal boutons in layer IV. However, in barrelless, the boutons are distributed within a larger tangential extent. Analysis of the distribution of boutons from neighboring thalamic neurons demonstrated the existence in layer IV of domains of high bouton density that in both strains equal the size and shape of individual barrels. We propose that the domains of high bouton density are at the basis of the whisker map in barrelless mice.
\end{abstract}

Key words: mice; barrel cortex; thalamocortical; adenylyl cyclase I; cortical map; biocytin

\section{Introduction}

In direct sensory pathways of the mammalian brain, axons projecting from thalamus to the primary sensory cortex have a stereotypic morphology. In the thalamus, they send collaterals to the reticular nucleus and subsequently ascend through the internal capsule to terminate in cortical layers VI and IV (Jones, 1985, 1986). Subcortically, the topological organization of thalamocortical axons (TCAs) undergoes distinct transformations (Adams et al., 1997). In cortex, the spatial segregation of TCAs underlies the functional parcellation (Blasdel and Lund, 1983; Humphrey et al., 1985; Jensen and Killackey, 1987a; Antonini et al., 1998, 1999). During development, sensory activity is an important factor in determining the spatial distribution of TCAs as was demonstrated after visual deprivation in cats (Antonini and Stryker, 1993) and whisker follicle denervation in rats (Jensen and Killackey, 1987b). Detailed studies of thalamic projections in the whisker-to-barrel pathway revealed that modifying neuronal transmission in cortex influences the spatial configuration of TCA during development. Blocking the activity through the NMDA receptor in the conditional NR1 knock-out affects TCA morphology but does not modify the cytoarchitectural differen-

\footnotetext{
Received March 24, 2006; revised July 27, 2006; accepted Aug. 14, 2006.

This work was supported by the Swiss National Science Foundation (310000-108246). We thank Laurent Tettoni for support throughout this study; Graham Knott for helpful comments on this manuscript; Nathalie Borrajo-Trapp and Nathalie Mueller for histology; and Gilles Bronchti, Vincent Castagné, Christel Genoud, Charles Quairiaux, Fabien Pichon, Aouatef Abaza, and Jan-Harry Cabungcal for helpful discussions.

Correspondence should be addressed to Egbert Welker, Département de Biologie Cellulaire et de Morphologie, Faculté de Médecine, Université de Lausanne, Rue du Bugnon 9, CH-1005 Lausanne, Switzerland. E-mail: Egbert.Welker@unil.ch.

DOI:10.1523/JNEUROSCI.1263-06.2006

Copyright $\odot 2006$ Society for Neuroscience $\quad$ 0270-6474/06/2610057-11\$15.00/0
}

tiation into barrels (Iwasato et al., 2000; Lee et al., 2005). Barrel formation is perturbed in several mouse lines: the knock-out for the metabotropic glutamate receptor 5 (mGluR5) receptor (Hannan et al., 2001), the monoamine oxidase A (MAOA)-deficient mouse (Cases et al., 1996), the GAP-43 (growth-associated protein 43) (Maier et al., 1999) and in the mouse mutant barrelless, which lacks functional adenylyl cyclase I (Welker et al., 1996; Abdel-Majid et al., 1998; Lu et al., 2003). Whereas a morphological analysis of the TCA axons is not available for the mGluR5 knock-out, in the other mouse lines lack of barrel formation is attributable to a disrupted clustering of TCA within barrel domains (Welker et al., 1996; Rebsam et al., 2002; McIlvain et al., 2003). Despite this altered arborization pattern of TCAs, a functional topographic map was demonstrated in SI of both MAOA and barrelless mice (Welker et al., 1996; Yang et al., 2001).

The aims of the current study are the following: (1) to characterize the arborization pattern of TCA in wild-type and barrelless mice to identify the level at which the mutation affected axonal morphology, and (2) to analyze whether the tangential distribution of the TCA boutons in barrelless mice can explain the establishment of a functional map of the sensory periphery. We reconstructed individual biocytin-labeled TCAs from thalamus to cortex and registered the spatial distribution of the axonal boutons that correspond to TCA synapses (White et al., 2004). We demonstrated that TCAs in barrelless are identical to those in wild type with respect to total axonal length and number of axonal boutons, but that the mutation affects the tangential extent of the axonal plexuses in layers IV and VI, confirming our previous report (Welker et al., 1996). Superposition of the distribution of axonal boutons from TCAs with cell bodies that are adjacent in the thalamus showed that within the cortex tangential domains 
are created with high density of axonal boutons that equal the size of individual barrels.

\section{Materials and Methods \\ Animals}

The barrelless mice (Welker et al., 1996) used in this study are from a breeding line that was started after the discovery of a mutation that had occurred in the normal (NOR) strain (i.e., mice bred for a standard pattern of mystacial vibrissas) (Van der Loos et al., 1984). Barrelless mice are homozygous for the BRL mutation that disrupted the gene coding for adenylyl cyclase type I on chromosome 11 (Welker et al., 1996; Abdel-Majid et al., 1998). In total, six NOR and 11 barrelless animals were used in this study. Mice of both strains were exposed to the same experimental procedures (see below) that were approved by the Office Vétérinaire Cantonal (Lausanne, Switzerland), in accordance with Swiss laws.

\section{Tracing and reconstruction of} thalamocortical axons

Adult females (age, 6-10 weeks) were anesthetized with Nembutal (60 mg of sodium pentobarbital/kg of body weight, i.p.) and placed in a stereotaxic frame. Glass pipettes filled with $2 \%$ biocytin in $1 \mathrm{~m}$ potassium acetate were lowered into the ventrobasal nucleus of the thalamus (VB) of the right hemisphere. Stimulationdependent neuronal activity was recorded through the pipette to identify the representation of the mystacial whisker follicles. Subsequently, the tracer was iontophoretically injected using a 150-200 nA positive current for 10-15 min (1 s on, $1 \mathrm{~s}$ off) according to the protocol of Pinault (1996). In most cases, the injection was made in the representation of follicle C2. After $24 \mathrm{~h}$, mice were reanesthetized with a lethal dose of Nembutal and were fixed via transcardial perfusion of $4 \%$ paraformaldehyde in phosphate buffer (0.1 M; pH 7.2). Brains were removed and postfixed for $2 \mathrm{~h}$ in same fixative after which they were placed in a $30 \%$ solution of sucrose overnight. Brains were cut coronally on a freezing microtome at $50 \mu \mathrm{m}$, and the sections were processed for the revelation of biocytin using the avidin-biotin reaction kit from Vectra stain. Sections were Nissl stained with methylene blue. For additional details on the histological procedure, see Dolleman-Van der Weel et al. (1994). Cortical areas were assigned using cytoarchitectonic criteria as described by Caviness (1975).

The number of labeled thalamic neurons was counted. A total of 35 NOR and 17 BRL axons were hand-drawn using an ordinary light microscope fitted with a drawing tube. These drawings were used to study the axonal trajectory and the principal axonal bifurcations in the somatosensory cortex. From these sets of axons, eight NOR axons (from four mice) and nine BRL axons (from three mice) were randomly assigned for threedimensional reconstruction using a Zeiss (Oberkochen, Germany) microscope equipped with a video camera (930XP 3CCD Color Video; Sony, Tokyo, Japan), a microcomputer-controlled stage, and a reconstruction program, Neurolucida (MicroBrightField, Williston, VT). Figure 1 gives the positions of the injection sites of these reconstructed axons.

Per mouse, two to six axons were reconstructed simultaneously from VB to the cortex over 10-25 sections. In the cortex, the entire axon was reconstructed, and the branch points (nodes) and endings were plotted. Axonal branches represent the axonal segments between two consecutive nodes. The branch was "incomplete" if, for any reason, its reconstruction was not possible. Branches with a length $\leq 4 \mu \mathrm{m}$ were not traced but their
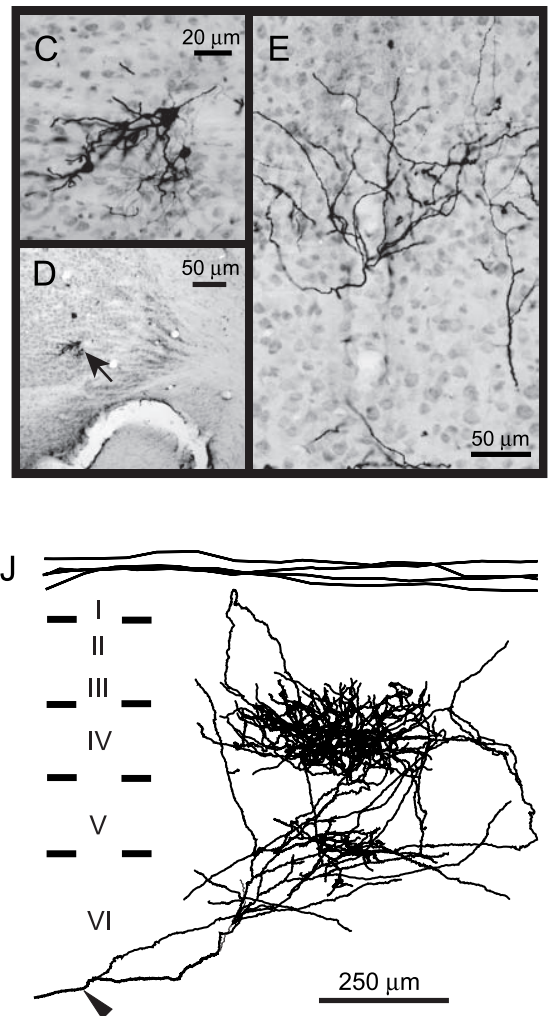

Figure 1. $\quad \boldsymbol{A}, \boldsymbol{B}$, Sites of biocytin injections in the VB of the mice that were used for quantitative analysis. Coronal sections 列 part),MD, medial dorsal nucleus; Pom, posterior complex, medial division; $R$, reticular nucleus of the thalamus. $C-J$, Illustration of axonal branches that carry axonal boutons ( $\boldsymbol{H}, \boldsymbol{I}$, arrows). The reconstruction of the axonal arborization is displayed in $\boldsymbol{J}$. The roman numerals indicate the cortical layers; the arrowhead indicates the point of entry into cortex.

position was identified. The position of the axonal boutons was also plotted. A correction $(2.66 \times)$ was applied for the shrinkage in the $z$-axis because of the histological procedure.

The NEUROLUCIDA data file was transferred to a UNIX station (Silicon Graphics, Mountain View, CA) for additional analysis using MAXSIM software (Tettoni et al., 1996).

\section{Measurements}

MAXSIM software enabled measurements of the entire axonal intracortical arborization and its components: the trunks and the tangential plexuses. Trunks were defined as parts of the axon that traversed the cortical layers and gave off branches in the two target layers: the upper layer VI and layer IV. The tangential plexus of layers I-IV are all the branches coming off by the axonal trunks in layers IV, III, II, and I. The tangential plexus of layers $\mathrm{V}-\mathrm{VI}$ is formed by the branches that course tangentially at the white matter (WM)/cortical border and at the VI/V interface. The following morphological parameters were determined.

The "neurometric" parameters. To characterize the axonal tree and the distribution of boutons, we measured the axonal length, number of axonal branches, and the position of each axonal bouton. Measurements of these parameters were made first on the entire intracortical arborization by selecting the axon at its cortical entrance (Fig. $2 \mathrm{~A}$, arrow). Using the Strahler centripetal ordering (Fig. $2 C$ ), the program further allows us to topologically characterize the axon. Two compartments were distinguished: a "transmission" compartment formed by the Strahler's orders 0 and 1 , and a "conduction" compartment formed by the sum of the orders 2, 3, and 4 (Fig. $2 B, C$ ).

To obtain the parameters of the layer IV tangential plexus, the en- 


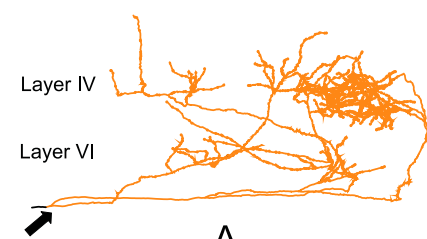

A
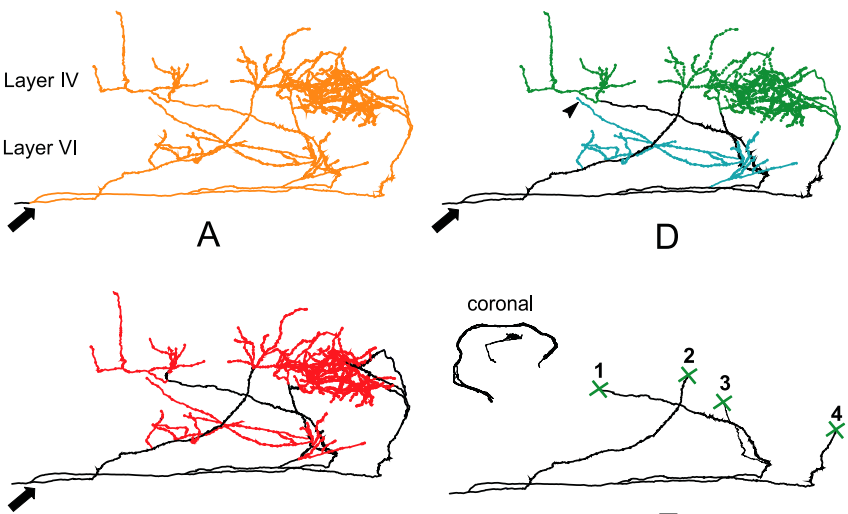

B

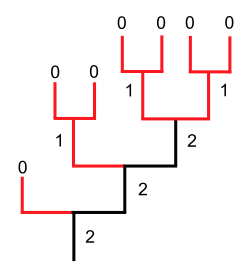

C

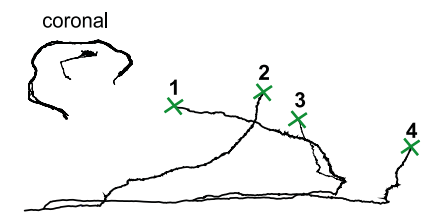

$\mathrm{E}$

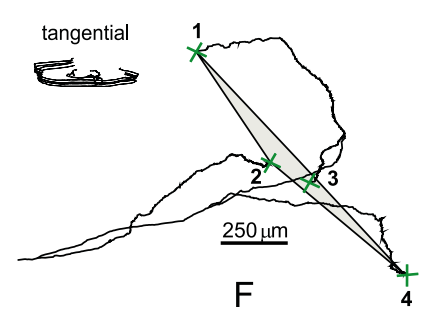

Figure 2. Axonal subdivisions used for the morphometric analysis as illustrated for a BRL axon. $A$, The intracortical arborization rendered yellow from the point where the axon enters the cortex (arrow). B, Transmission compartment (red) and conduction compartment (black). C, Topological Strahler ordering is centripetal, coding each terminal branch 0 and proceeding toward the root of the branch. When two branches of the same order meet, the order number of the proximal branch is incremented by 1 ; otherwise, the largest order is used. The transmission compartment is formed by the Strahler's orders 0 and 1 and contains most of the tangential plexuses rich in boutons. The conduction compartment is the sum of Strahler's orders 2,3, and 4 , and corresponds to the axonal trunks. $\boldsymbol{D}, \boldsymbol{E}$, Green crosses in $\boldsymbol{E}$ define the axonal plexus in layer IV and above ( $\boldsymbol{D}$, green). The branches running tangentially in the infragranular layers were selected at their emergence from the radial trunks and formed together the tangential plexus in layers V-VI ( $\boldsymbol{D}$, blue). Some terminal branches of the V-VI plexus reached layer IV without giving arborizations in this layer $(\boldsymbol{D}$, arrowhead). $\boldsymbol{F}$, The axonal trunk was turned in a tangential position; the marked points $1-4$ were unified in a convex polygon to calculate the area of entry into layer IV. The insets in $\boldsymbol{E}$ and $\boldsymbol{F}$ indicate the orientation of the reconstructed axons.

trance of the trunk in this layer was identified (Fig. $2 E$, green crosses). For the $\mathrm{V}-\mathrm{VI}$ plexus, the tangential oriented infragranular branches (Fig. $2 \mathrm{E}$, blue) were selected at the emergence of their trunk. The points at which the axonal trunks entered layer IV were further used to determine the "entrance surface" of the axon in layer IV (see below).

The "surface" parameters. These parameters are two-dimensional and represent the areal extent of the axon projection in a plane tangential to the pial surface overlying the representation of the large mystacial whisker follicles (Woolsey and Van der Loos, 1970). In this plane, we determined the entrance surface of the axon in layer IV (Fig. $2 F$ ) and analyzed the distribution of axonal boutons of the tangential plexuses in layer IV and layer VI using a grid consisting of $20 \times 20 \mu \mathrm{m}$ squares. In each square, the density of boutons was calculated. The total surface area is the sum of squares in which one or more boutons were present. For additional analysis, we used five density ranges: $(0,1],(1,2],(2,4],(4,8]$, and $(8,64]$.

\section{Statistics}

Statistical analyses were performed using the SAS software package (SAS Institute, Cary, NC) and according to SAS/STAT user's guide, version 6. The distribution of parameters was tested for normality, and rank transformations were used if necessary. The differences were considered to be statistically significant at $p \leq 0.05$.

The morphometric parameters were compared using a multivariate ANOVA (SAS/STAT user's guide, version 6; GLM procedure). A factor analysis (FACTOR procedure) was used to obtain a global image of the

NOR

BRL

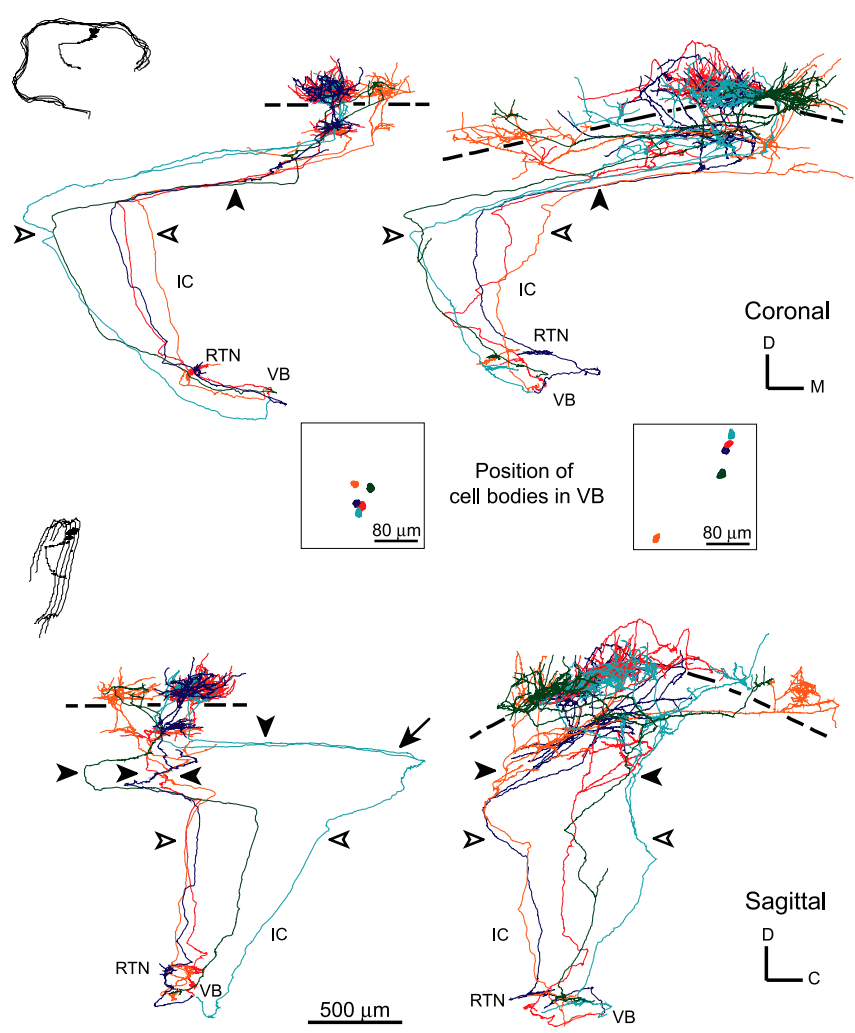

Figure 3. Display of five thalamocortical axons in NOR and in BRL to illustrate the trajectory from VB to the somatosensory cortex and maintenance of thalamic neighborhood relationship in cortical termination in both strains. The NOR axons are represented in the left column; the BRL axons are represented in the right column. In the top part, axons are displayed in a coronal plane, turned such that the radial orientation of the axonal arbor is vertical; in the bottom part, axons are displayed in a sagittal plane (insets to the left, indicate the orientation of the reconstructed axons). Boxes, Spatial relationship of the labeled neurons in the VB, as displayed in a coronal plane using the same color coding as for the axons. Filled arrowheads indicate entry in the cortex; open arrowheads indicate the point where the axons leave the internal capsule (IC) to enter subcortical WM; line segments, the border between layers IV and V; all axons gave off collaterals into the reticular thalamic nucleus (RTN). Note that despite the high divergence of the cortical termination in BRL, the cellular neighborhood in the thalamus was respected at the cortical level. In these reconstructions, the boutons are not displayed. The scale bar at the bottom pertains to NOR and BRL (coronal and sagittal).

interdependencies between the parameters. Only factors with an eigenvalue $\geq 1$ were considered to well characterize the variability inside a tangential plexus. A regression model was used (GLM and REG procedures) to study the hypothesis of homogeneity of slopes between strains for each layer and between layers inside each strain.

A $\chi^{2}$ test (FREQ procedure) was used to tests the differences between the frequency distribution of the number of terminals reached after a given number of nodes in NOR and BRL.

\section{Results}

Neuronal labeling and subcortical trajectory

Subcortical axonal trajectory is common in NOR and BRL

Biocytin injections were made in the VB of the thalamus where the large mystacial whisker follicles are represented (Fig. $1 A, B, D)$. For both NOR and BRL, injections situated more dorsally resulted in labeled cells whose axons gave arborization in SI, whereas those situated more ventrally, gave arborizations in SI and SII. The labeled axons were seen to arise directly from the soma or from a dendrite close to its emergence from the soma.

The study of the hand-drawn TCAs from $\operatorname{NOR}(n=35)$ and 
BRL $(n=17)$ showed that the subcortical trajectory is very similar in both strains (Fig. 3). Inside VB, the TCA change direction several times at variable distances from the cell body before arriving at the VB border. TCAs cross the thalamic reticular nucleus (RTN) parallel to each other. In this nucleus, the axons give off collaterals (1-4 per axon; mean number, 1.9) that form a dense plexus oriented perpendicular to the trajectory of the labeled axons. Leaving the RTN, TCAs enter the internal capsule in which axons follow diverging trajectories through the striatum. Figure 3 illustrates this divergence both in a coronal and sagittal plane. It shows that axons from neurons that are close neighbors in $\mathrm{VB}$ do not maintain this proximity within their trajectory to the somatosensory cortex. On reaching the subcortical WM, some TCAs run for long distances at the border with layer VI; other axons enter the cortex and travel tangentially for some distance in layer VI. The cortical entrance and the eventual axonal branching at the WM/VI interface are, in all cases, within the boundaries of the somatosensory cortex.

Using the set of computer-reconstructed axons, we found that the subcortical trajectory of TCA of NOR axons has a mean length of $3.4 \pm 0.4 \mathrm{~mm}$ and is not different from that of BRL axons $(3.7 \pm 0.4 \mathrm{~mm})$.

Progressive convergence in NOR

In NOR, the majority (32 of 35) of labeled axons restrict their cortical entrance to the territory of three neighboring columns, including the "target" barrel. However, some axons (3 of 35) enter SI at its border with SII, with occipital (Fig. 3, NOR, sagittal view, arrow) or with motor cortex. These axons curve at 90 or $180^{\circ}$ and travel inside layer VI to reach the column of their target barrel.

Of the 35 axons, only one enters the cortex within the appropriate barrel column. Of the 34 axons, 19 converge (with one or more of their trunks) to the appropriate column within layer VI; 10 others direct their trunks toward the appropriate barrel column within layer $\mathrm{V}$ or at its border with layer IV. One axon corrects the trajectory of one of its trunks at the level of layer IV, whereas two axons use the supragranular layers for turning into the appropriate barrel column. The trajectories of these 33 axons illustrate the trend to progressively converge toward the appropriate barrel column from layer VI upward. Two axons do not converge with their trunks into a single barrel column; their trunks enter two barrels, where they give raise to an extensive terminal plexus in one barrel and give off some terminal branches in the other.

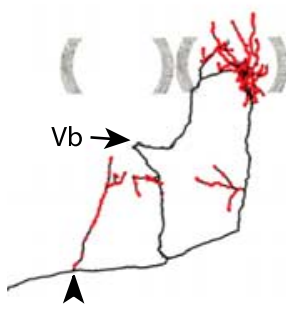

A

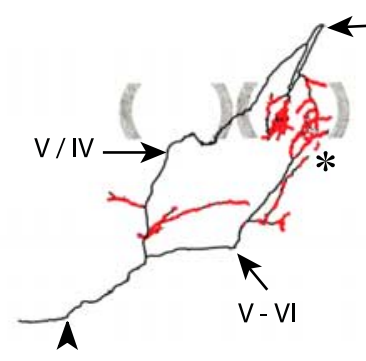

B

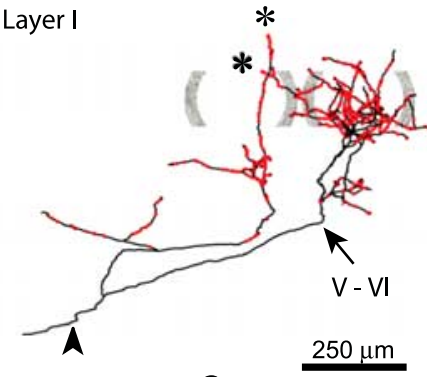

C

Figure 4. Intracortical trajectories of three thalamocortical axons in NOR. Roman numerals indicate cortical layers; arrowheads, point of entry into cortex; arrows, the change of orientation of axonal branches within the cortex; red dots, the boutons. Barrel outlines are rendered gray and were drawn from the Nissl-stained section. Not all axonal branches in layer IV are reproduced here. Axons are displayed in a coronal view with the pial surface up. The axon in $\boldsymbol{A}$ has infragranular collaterals in two neighboring barrel columns. One collateral has its origin at the WM/VI border and does not reach layer IV; the other redirects its trajectory in layer $\mathrm{Vb}$ and enters the neighboring barrel, where it joins the third collateral. These collaterals enter the barrel from its peripheral border. The axon in $\boldsymbol{B}$ has its first branching point in layer Vl: one collateral ascends radially within a barrel column and switches to a neighboring column at the border between layers IV and V; it crosses layer IV without branching and reaches layer I, where it makes a U-turn. On reentry in layer IV, it gives terminal branches in the same barrel as the other two infragranular collaterals, a trunk and a terminal 0 branch. The axon in C gives two collaterals at its entry into the cortex. Both collaterals reach layer IV but in adjacent barrels, where one gives rise to a rich plexus of terminal branches (with branches crossing the barrel boundary), whereas the other terminates without branching. The scale bar in $\boldsymbol{C}$ pertains to $\boldsymbol{A}-\boldsymbol{C}$. Asterisks indicate infragranular branches (order 0 ) that enter layer IV without giving off other branches.

A

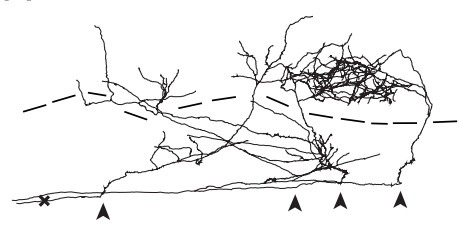

C

Figure 5. Intracortical trajectories of three thalamocortical axons in BRL mice. Roman numerals indicate cortical layers; arrowheads, points of entry into cortex; dots, entries of the trunk in layer IV; line segments, the border between layers IV and V. Boutons are not plotted. Axons are displayed in a coronal view with the pial surface up. Axon in $\boldsymbol{A}$ has its first branching point in the WM: the two axonal collaterals run parallel within WM where one of them has a small branch ( $4 \mu \mathrm{m}$ long; at cross). This collateral does not branch further before entering the cortex. The other collateral travels for a long distance at the WM/VI border, where it gives off numerous branches. These branches reach layer IV independently from each other at great distances, and they give a variable number of layer IV branches. The axon in $\boldsymbol{B}$ has the first branching point at the layer VI-WM interface. One collateral travels between WM and layer $\mathrm{VI}$, and the other one gives a dense plexus between layers $\mathrm{V}$ and $\mathrm{VI}$ and several main branches that ascend and enter layer IV in three distinct regions at great distances from each other. One of these branches travels a distance of $\sim 2000$ $\mu \mathrm{m}$ in layer Vb to reach layer IV. In layer IV, this axon forms multiple terminal plexuses that differ in their branch density. The axon in Chas two branch points at the limit between the WM and layer VI that produce three collaterals that terminate in layer $V$ or at the limit between the WM and the layer VI. The main axon ascends to layer IV and gives two trunks in layer Vb. One of these trunks branches in layer IV and reaches layer I, where it gives another branching point whose daughter branches make a U-turn to descend in layer IV. Even if the trunks enter in the same region of layer IV, their arborizations in layer IV are highly divergent. The scale bar in $\boldsymbol{C}$ pertains also to $\boldsymbol{A}$ and $\boldsymbol{B}$.
In layer VI and in layer IV, NOR axons give off branches that give rise to bouton-rich plexus (Fig. $4 A-C$ ). In general, the infragranular plexus extends into several barrel columns, whereas the plexus in layer IV is highly concentrated in a single barrel. Two of the 35 analyzed axons terminated in more than one barrel; one of these is illustrated in Figure 4C. The layer VI plexus is essentially formed by branches that run tangentially in upper VI and that originate from radial trunks that ascended to layer IV (Fig. 4A,B). 


\begin{tabular}{|c|c|c|c|c|c|c|}
\hline & \multicolumn{2}{|c|}{ Intracortical arborization } & \multicolumn{2}{|l|}{ Layers I-IV } & \multicolumn{2}{|l|}{ Layers V-VI } \\
\hline & NOR & $B R L$ & NOR & $\mathrm{BRL}$ & NOR & $\mathrm{BRL}$ \\
\hline \multicolumn{7}{|l|}{ Intracortical arborization } \\
\hline Total axonal length $(\mathrm{L})$ in $\mu \mathrm{m}$ & $17,022 \pm 8057.7$ & $21,555.7 \pm 5632.1$ & $12,274.5 \pm 5858.7$ & $14,152.8 \pm 4777.7$ & $2921 \pm 2190.2$ & $3744.9 \pm 3036.7$ \\
\hline Total number of boutons (B) & $1233.9 \pm 836$ & $1336.3 \pm 590.6$ & $1057.7 \pm 679.1$ & $1112 \pm 464.9$ & $175 \pm 207.2$ & $198.3 \pm 192$ \\
\hline Total number of branches (BR) & $209 \pm 102.3$ & $196.4 \pm 40.7$ & $167 \pm 80.3$ & $154.1 \pm 38.2$ & $33 \pm 28.5$ & $28.5 \pm 19.9$ \\
\hline$D=$ No. boutons $/ 100 \mu \mathrm{m}$ axonal length & $6.7 \pm 1.9$ & $5.9 \pm 1.4$ & $8 \pm 2.2$ & $7.9 \pm 1.3$ & $4.7 \pm 2.1$ & $3.9 \pm 2.5$ \\
\hline \multicolumn{7}{|l|}{ Transmission compartment $(0+1)$} \\
\hline $\mathrm{LT}=\mathrm{L} 0+\mathrm{L} 1$ in $\mu \mathrm{m}$ & $13,940.3 \pm 6632.1$ & $16581.7 \pm 5442.5$ & $11,260.6 \pm 5328.9$ & $12,809.6 \pm 4216.5$ & $2624.7 \pm 2064.1$ & $3362.3 \pm 2759.3$ \\
\hline $\mathrm{B} 0+\mathrm{B} 1$ & $1195.9 \pm 812$ & $1289.1 \pm 570.5$ & $1026.4 \pm 656.6$ & $1076.8 \pm 451.9$ & $169.1 \pm 205.2$ & $193.9 \pm 190.3$ \\
\hline $\mathrm{BRO}+\mathrm{BR} 1$ & $165.4 \pm 77.9$ & $155.7 \pm 31.7$ & $136.4 \pm 62.9$ & $127.6 \pm 29.4$ & $29.1 \pm 25.1$ & $25.9 \pm 16.1$ \\
\hline$L 0+L 1 / L(\%)$ & $81.7 \pm 3.8$ & $75.6 \pm 7.3$ & $91.9 \pm 1.5$ & $90.9 \pm 2.9$ & $91.6 \pm 10.7$ & $92.5 \pm 11.1$ \\
\hline $\mathrm{B} 0+\mathrm{B} 1 / \mathrm{B}(\%)$ & $97.0 \pm 1$ & $96.6 \pm 1.2$ & $97.3 \pm 1$ & $96.9 \pm 1.4$ & $96.7 \pm 3.8$ & $94.6 \pm 7.4$ \\
\hline $\mathrm{BRO}+\mathrm{BR} 1 / \mathrm{BR}(\%)$ & $79.5 \pm 1.7$ & $79.3 \pm 0.9$ & $82.5 \pm 2.9$ & $83 \pm 3.2$ & $91.5 \pm 9.3$ & $92.8 \pm 7.5$ \\
\hline \multicolumn{7}{|l|}{ Conduction compartment $(2+3+4)$} \\
\hline $\mathrm{LC}=\mathrm{L} 2+\mathrm{L} 3+\mathrm{L} 4$ in $\mu \mathrm{m}$ & $3019.6 \pm 1471.1$ & $4988.4 \pm 1101.7$ & $1014 \pm 542.1$ & $1342.6 \pm 680.8$ & $298.8 \pm 320.3$ & $398.1 \pm 580.4$ \\
\hline $\mathrm{B} 2+\mathrm{B} 3+\mathrm{B} 4$ & $38 \pm 25$ & $48.3 \pm 26.8$ & $31.4 \pm 23.6$ & $35.4 \pm 21.5$ & $5.9 \pm 6$ & $5 \pm 6.5$ \\
\hline $\mathrm{BR} 2+\mathrm{BR} 3+\mathrm{BR} 4$ & $43.3 \pm 24.1$ & $40.6 \pm 9.3$ & $31.4 \pm 18.4$ & $25.7 \pm 10.7$ & $3.9 \pm 4.4$ & $2.7 \pm 2.8$ \\
\hline $\mathrm{L} 2+\mathrm{L} 3+\mathrm{L} 4 / \mathrm{L}(\%)$ & $18.1 \pm 3.9$ & $24.4 \pm 7.3$ & $8.1 \pm 1.5$ & $9.1 \pm 2.9$ & $8.6 \pm 10.5$ & $7.8 \pm 12.1$ \\
\hline$B 2+B 3+B 4 / B(\%)$ & $3 \pm 1$ & $3.5 \pm 1.1$ & $2.7 \pm 1.0$ & $3.1 \pm 1.4$ & $3.3 \pm 3.8$ & $2.4 \pm 2.9$ \\
\hline $\mathrm{BR} 2+\mathrm{BR} 3+\mathrm{BR} 4 / \mathrm{BR}(\%)$ & $20.4 \pm 1.5$ & $20.5 \pm 1.1$ & $18 \pm 3.1$ & $16.2 \pm 2.8$ & $8.5 \pm 9.3$ & $7.2 \pm 7.5$ \\
\hline Tangential surfaces $\left(\mu \mathrm{m}^{2}\right)$ & & & $63,880 \pm 26,840$ & $173,760 \pm 55,000$ & $54,057.1 \pm 28,447.3$ & $78,342.9 \pm 75,960.5$ \\
\hline Surface of entrance in layer IV $\left(\mu \mathrm{m}^{2}\right) \mathrm{SE}$ & $2834.5 \pm 3172.1$ & $88,127 \pm 111,554$ & & & & \\
\hline Mediolateral extent ( $\mu \mathrm{m})$ & & & $441.7 \pm 129.5$ & $1360.4 \pm 609.1$ & $382.1 \pm 224$ & $1119.5 \pm 548.3$ \\
\hline
\end{tabular}

Values are expressed as mean \pm SD.

In two cases, we identified branches that arise from nodes at the WM/VI border climb in upper VI where they contribute to the infragranular plexus (Fig. $4 B$ ).

Progressive divergence in $B R L$

At the cortical level, BRL axons are highly divergent (Fig. 3) and do not enter SI within a three barrel column area as described above for NOR. Of the 17 hand-drawn BRL axons, 12 enter SI with several, highly divergent, branches (Fig. 5A) that climb up to layer IV. In addition to these long, bouton-bearing branches, we also notice a large number of very short axonal branches $(<4$ $\mu \mathrm{m})$ that are formed by the axon at the level of the border between the white matter and layer VI (Fig. $1 F$ ). However, four axons enter SI with a single trunk, but bifurcate in the infragranular layers and give off branches that run $\sim 1.5 \mathrm{~mm}$ tangentially inside layer $\mathrm{Vb}$, before entering layer IV (Fig. $5 B$ ). One axon approaches layer IV with a single axonal trunk that bifurcates just before entering (Fig. 5C).

The number of branches by which a TCA enters layer IV in barrelless is twice that of NOR [BRL, $5.4 \pm 2.2(\mathrm{SD})$; NOR, $3.3 \pm$ $1.4(\mathrm{SD}) ; p<0.005]$. In addition, the surface of entrance of these branches is 31 times larger than in NOR (Table $1, p=0.001$ ). Several of these branches are given off at the border between white matter and layer VI or during the intracortical trajectory of the axon. The result of this branching pattern is a progressive divergence of the axonal arborization while reaching layer IV, which is in contrast with the increase convergence by which TCA approaches layer IV in NOR.

As in NOR, TCA in barrelless give rise of axonal bouton-rich plexuses in layers IV and VI. However, inside the layer IV plexus in barrelless, axonal branches seem not to encounter a spatial restriction, which adds another factor to the total divergence of the axonal arbor.

Despite the divergence, in their cortical termination, BRL axons conserve their topographic relationship as found in the thal-

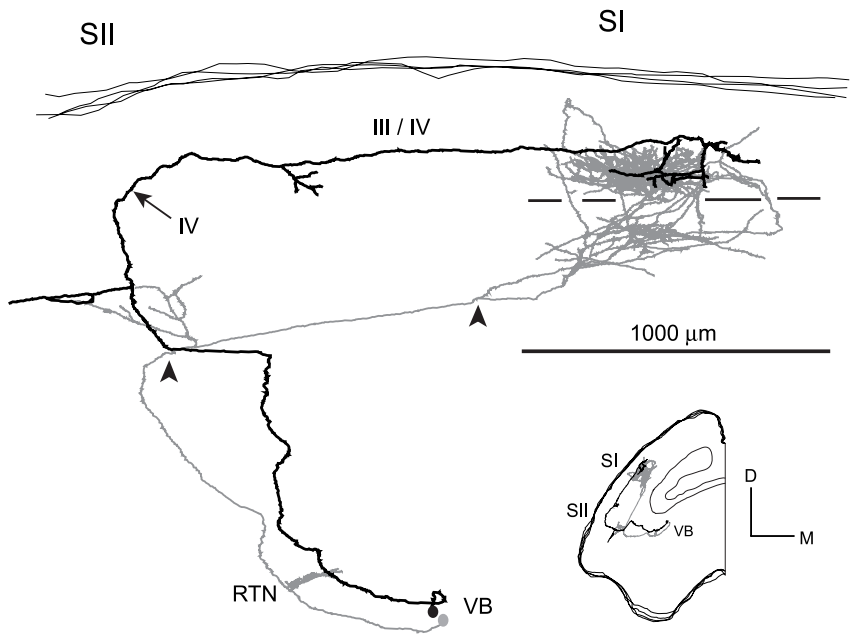

Figure 6. BRL axons that arborize in SII. Roman numerals indicate cortical layers; arrowheads, point of entry into the cortex; arrows, the change of orientation of axonal branches within the cortex; line segments, the limit between layers IV and V. For the sake of clarity, one axon was depicted in black and another in gray. The boutons are not plotted. The axons are displayed in a coronal view with the pial surface up (inset for orientation). The BRL axons reach $\mathrm{SI}$ in different ways. After having given a collateral in SII, the gray axon continues in the WM to curve into SI. The black axon enters SII, gives a tangential branch at the interface V/VI, climbs superficially, makes a $90^{\circ}$ turn in layer IV, and then travels $\sim 1500 \mu \mathrm{m}$ inside the cortex to join the gray axon in SI. Note that the cell bodies of the above axons are close together in the VB. RTN, Reticular thalamic nucleus.

amus (Fig. 3, BRL). An example is depicted in Figure 6. In this BRL mouse, the biocytin injection was performed more ventrally in the thalamus and TCA made branches in both SI and SII. The biocytin-labeled cell bodies were close together in VB. One axon, instead of reaching SI by traveling at the WM/VI interface, entered in the cortex at the SI/SII border and gave branches in layer VI of the SII. It subsequently traveled in layer III for $\sim 1500 \mu \mathrm{m}$ to reach the same region in SI as its VB neighbor. 
A

\begin{tabular}{|c|c|c|c|c|}
\hline & \multicolumn{2}{|c|}{ NOR } & \multicolumn{2}{|c|}{ BRL } \\
\hline & Factor 1 & Factor 2 & Factor 1 & Factor 2 \\
\hline LT & 0.961 & 0.211 & 0.987 & 0.054 \\
\hline LC & 0.935 & -0.017 & 0.103 & 0.877 \\
\hline $\mathbf{V}$ & 0.929 & 0.221 & 0.966 & -0.034 \\
\hline 54 & 0.814 & 0.352 & 0.883 & -0.246 \\
\hline SE & 0.155 & 0.978 & -0.238 & 0.781 \\
\hline eigenvalue & 3.63 & 0.89 & 2.81 & 1.38 \\
\hline Proportion & $72.6 \%$ & $17.8 \%$ & $56.3 \%$ & $27.6 \%$ \\
\hline
\end{tabular}

B

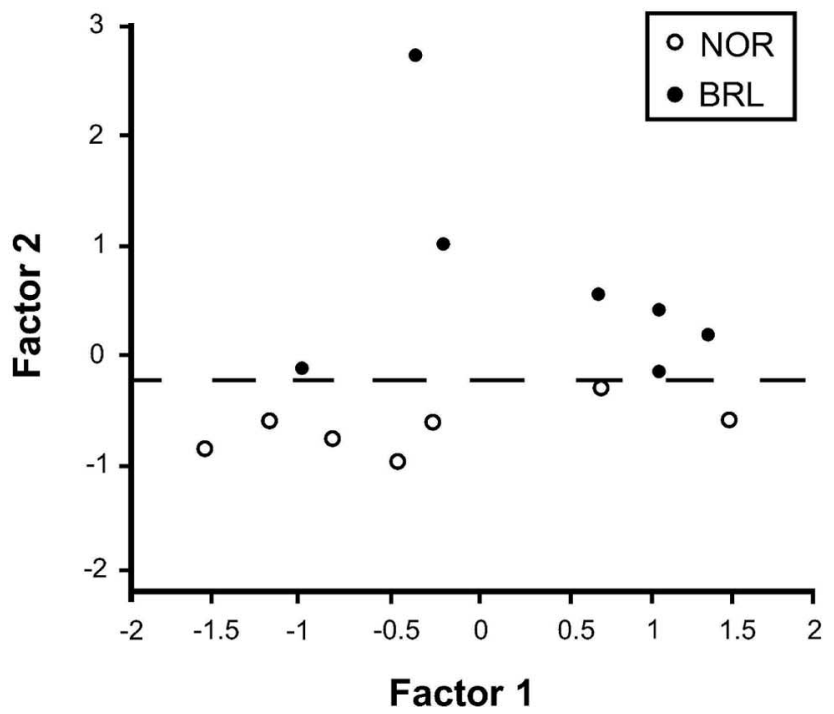

Figure 7. A, Table showing the correlations of factor 1 and factor 2 with the five parameters LT, LC, B, S4, and SE. Significant correlations are in gray; the eigenvalues and the proportion of the variability explained by each factor are given at the bottom. Factors were determined for NOR and BRL samples separately. LT, Length of the transmission compartment of the whole intracortical arborization; $L C$, length of the conduction compartment of the whole intracortical arborization; $B$, number of boutons of the whole intracortical arborization; $\$ 4$, total tangential extent of the axons in layers I-IV; SE, surface of entrance of the axonal trunks in layer IV. $\boldsymbol{B}$, Graphical display of the value of factors 1 and 2 for each individual axon. Line segments indicate the level at which factor 2 segregates the axons from NOR mice (open dots) and the BRL axons (black dots). Statistical analysis proved this segregation to be significant ( $p=0.001$ ).

\section{Quantitative analysis of individual axons}

Analysis of the intracortical arborizations

Table 1 lists the quantitative parameters used to analyze the differences between NOR and BRL axons. On average, the intracortical arborizations of TCA in NOR mice have a length $(L)$ of 17.0 $\mathrm{mm}$ (SD, $8.1 \mathrm{~mm})$, carry 1234 (SD, 836) boutons (B), and are formed by 209 (SD, 196) branches (BR). In the granular and supragranular layers, these axons spread over a mean tangential surface (S4) of $0.083 \mathrm{~mm}^{2}$ (SD, 0.026), the intracortical arborization of BRL axons have a tendency to be longer $(21.5 \pm 5.6 \mathrm{~mm})$ than those in NOR, but possesses the same mean number of boutons (1336 \pm 590$)$ and mean number of branches $(196 \pm 41)$. The difference in length is mainly attributable to a $40 \%$ increase of the conduction compartment in BRL. The tangential surface $\mathrm{S} 4$ in BRL is $0.17 \mathrm{~mm}^{2}$ (SD, $\left.0.055 \mathrm{~mm}^{2}\right)$, twice that in $\operatorname{NOR}(p<$ 0.002).

The differences between NOR and BRL axons were statistically analyzed using a one-way multivariate analysis and a factor analysis on the combination of the following five parameters: $\mathrm{B}$

\section{Frequency distribution \\ of the number of terminal segments}

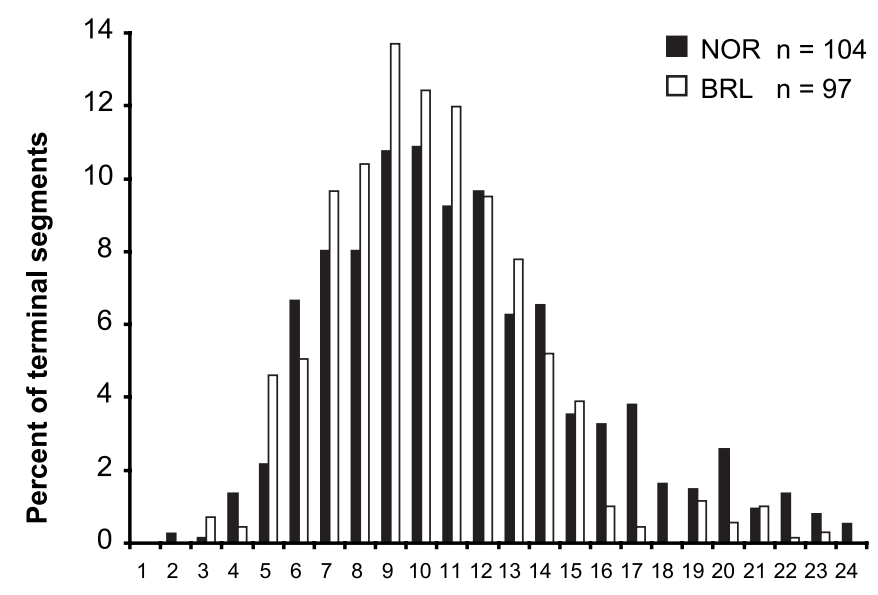

Number of nodes

Figure 8. Frequency distribution of the number of terminal branches reached by a hypothetical action potential after traversing a given number of nodes within the intracortical arborization of NOR and BRL axons. The two distributions are of similar shape, but the BRL distribution is significantly shifted to the left $\left(\chi^{2}\right.$ test, $\left.p<0.001\right)$. As a consequence, in BRL axons, action potentials will reach the terminal axonal branches by traversing a smaller number of bifurcations.

represents the total number of boutons of the entire intracortical arbor (Fig. $2 \mathrm{~A}$, the yellow portion of the axon); LT represents length of the transmission compartment of the cortical arborization (all the branches of order 0 and 1 found mostly in the layers I-IV and V-VI) (Fig. 2 , the red portion of the axon); LC is the length of the conduction compartment of the intracortical arborization (the axonal trunks plus all the branches of order 2, 3, and 4 inside the tangential plexuses) (Fig. $2 \mathrm{~B}$, blue); SE is the entrance surface of the axonal trunks in layer IV (Fig. 2 F); S4 is the tangential surface of the axon inside layers I-IV.

LC and SE parameters characterize the part of the axon by which it reaches layer IV. The parameters V, S4, and LT characterize the tangential plexuses where most of the boutons and the 0 and 1 order branches are found.

A one-way multivariate analysis on the combination of the five parameters LT, LC, B, S4, and SE demonstrated an important "strain effect" ( $p=0.009)$. To analyze the interdependencies of the five parameters, a factor analysis was performed on NOR and BRL axons as a single sample. It demonstrated that in both strains individual TCAs are characterized by (only) two factors. Figure $7 \mathrm{~A}$ indicates how the five parameters correlate to these two factors in NOR and in BRL. For NOR axons, a single factor ("factor 1 ") was sufficient to explain $72 \%$ of the variability with an eigenvalue of 3.63. This factor comprises four of the parameters, except SE that was found to be part of second factor ("factor 2") responsible for only $18 \%$ of the variability and which had no statistical significance (eigenvalue $<1$ ). In BRL axons, factor 1 covered only $56 \%$ of the variability and the analysis identified a significant contribution of factor 2 comprising $27 \%$ of the variability with an eigenvalue of 1.38 . For these axons, factor 1 highly correlated with B, LT, and S4, and factor 2 correlated with LC and SE. This multivariate analysis therefore indicates that the strain difference between TCA of NOR and BRL is expressed by two parameters, LC and SE, whereas other parameters seem to have been unaf- 
A. Whole intracortical arborization

B. Tangential plexuses
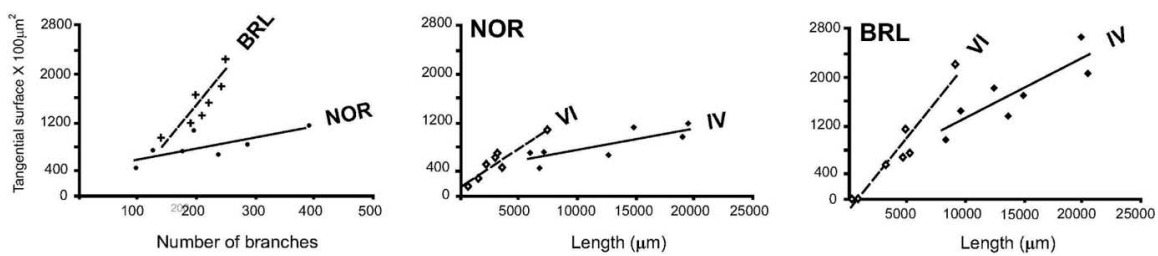

Figure 9. Correlations between the parameters characterizing NOR and BRL axons. $\boldsymbol{A}$, The tangential extent of NOR and BRL axons inside layer IV is plotted as a function of their total number of intracortical branches (i.e., axonal segments between consecutive nodes). Data on NOR axons are indicated by dots; BRL axons, by crosses. In both strains, the two parameters are significantly correlated ( $p=0.009$ for both NOR and BRL), but for the same number of branches BRL axons span a significantly larger cortical surface than NOR axons (strain effect, $p<0.001$ ). For the same increase in the number of branches, BRL axons increased their tangential surfaces more than NOR axons (analysis on heterogeneity of the slopes of the regression lines of NOR and $\mathrm{BRL}, p=0.005)$. The tangential extent of NOR $(\boldsymbol{B})$ and $\mathrm{BRL}(\boldsymbol{C})$ axons inside layer IV or VI is plotted as a function of the total axonal length in these layers. For the same increase of total axonal length, NOR axons increased their tangential surface in the infragranular layers more than inside layer IV (layer and length effect or comparison between NOR I-IV and NORV-VI slopes, $p=0.01$ ). This slope difference also exists in BRL $(p=0.008)$.

fected by the mutation. Figure $7 B$ is a graphical representation of the distribution of these two factors and demonstrates how factor 2 segregates the group of BRL axons from those of NOR.

Because LC represents the length of the conduction compartment, we analyzed whether the strain difference for this parameter also affects the geometrical complexity of the thalamocortical axon. For this, we determined for each terminal branch (order 0) the number of nodes (axonal bifurcations) that an action potential has to pass before reaching it from its entrance in the cortex. Subsequently, terminal branches were classed according to the number of nodes, and Figure 8 displays their frequency distribution. Statistical analysis identified that this distribution is significantly different between the two strains: terminal branches in BRL axons are reached by passing fewer nodes than in $\operatorname{NOR}\left(\chi^{2}\right.$ test, $p<0.001)$. This shows that thalamocortical axons in BRL have a longer conduction compartment (LC) but have a simpler branching pattern than those in NOR.

We further analyzed the strain difference in the geometry of the TCAs by testing the correlation between the branch number and the size of their tangential spread. As shown in Figure $9 A$, in both strains a significant linear correlation between the two parameters is found. However, the correlation is significantly different between the two strains $(p=0.005)$ : compared with NOR, the same variation in branch number produces in BRL a significantly larger increase in tangential spread in layer IV.

The multivariate statistical analyses described above identifies that the BRL mutation affects the parameters characterizing the conduction compartment of the thalamocortical axon as well as the geometry of the axonal arborization. Therefore, some of the results of the univariate analysis are worth mentioning (Table 1).

The total length of the conduction compartment in BRL was 1.6 times longer as in NOR (Table $1, p=0.02$ ), and its surface of entrance was 31 -fold greater (Table $1, p=0.001$ ). No statistical differences were found for the transmission compartment (Table 1). Significant strain differences were detected for the extent of the tangential surface (S4), which was twice as large in BRL as in $\operatorname{NOR}(p=0.002)$. Also, the mean mediolateral extent of the axons, as determined in the coronal plane, showed a significant difference by being three times larger in BRL than in NOR (BRL, $1.3 \mathrm{~mm}$; NOR, $0.4 \mathrm{~mm} ; p=0.002$ ).

Also, the correlation function between the tangential surface of the axonal plexus and the axonal length is significantly different between the strains (Fig. 9B, C). This correlation function is, in both strains, significantly different between the plexus in layers I-IV and that in layer V-VI: for NOR, $p<0.01$; and for BRL, $p<0.008$.

\section{Distribution of bouton densities areas in layers $I-I V$}

As mentioned above, by using the same axonal length, number of boutons, and axonal branches, BRL axons made surfaces twice as large as those of the NOR axons. Therefore, the density of boutons per unit of tangential surface was greater for NOR axons than for BRL axons ( $p$ value for mean density analysis). In this final part of the analysis, we studied the distribution of axonal boutons within the plexuses in layer IV and in layer VI. For this, we calculated the density of boutons per $20 \times 20$ $\mu \mathrm{m}$ area of cortical surface and analyzed the distribution within a series of density ranges. Figure 10 displays the density distribution for three NOR and three BRL axons as well as the relative distribution of the density classes per strain. In NOR, the TCA distributes axonal boutons in a restricted and compact part of layer IV, with a large area of high bouton density range situated in the center of the axonal plexus. In BRL, the total cortical area receiving TCA boutons in layer IV is larger, but the area covered by high bouton density is distributed throughout the plexus, not forming a compact area.

Performing a one-way univariate statistical ANOVA on the absolute values of the density areas inside layer IV confirmed the strain difference and showed that, in BRL, the surface with the lowest bouton density $(0,1]$ was more than twice as large as in NOR $(p<$ 0.001 ) and formed $33 \%$ of the total surface in NOR and $43 \%$ in BRL (Fig. 10G-I). Surfaces of intermediate density $(1,2],(2,4]$, and $(4,8]$ were approximately twice as large in BRL than in NOR $(p<0.001 ; p=0.006 ; p=0.01)$, and each of them represented $14-20 \%$ of the total tangential surface in both NOR and BRL. A smaller part of the total tangential extent of the axons was occupied by surfaces with a high bouton density $(8,64)(20 \%$ in NOR vs $6 \%$ in BRL). This analysis revealed a disproportionate distribution of axonal boutons in the layer IV tangential plexus of BRL axons that resulted in a twofold larger area of low and medium densities as compared with NOR. The high densities $(8,64]$ form a compartment that in absolute value was of similar size in both strains.

Performing the same statistical analysis on the density areas formed by the boutons of the plexus in layers V-VI showed no significant differences between strains (Fig. 10J-L).

Realizing that cortical compartmentalization is not attributable to a single TCA, but several hundreds, we analyzed the distribution of boutons formed by a pair of reconstructed axons that have their cell bodies close to each other in VB. Figure $11 \mathrm{~A}$ depicts two NOR axons that reached the same cortical barrel and that together formed a maximum density peak of 39 boutons $/ 400$ $\mu \mathrm{m}^{2}$. In BRL, the two axons tended to form their high-density surfaces in the same cortical region (Fig. $11 B$ ) and reached a maximal density that was similar with those formed by NOR axons. In BRL, this area of high density is surrounded by a large area of low density. Adding a third BRL axon to this analysis resulted in a even more pronounced delineation of a high-density area (Fig. 11C) that is only slightly larger in its tangential dimen- 
sion than the barrel-shaped area in NOR. The addition of the bouton distribution of two additional TCAs shows the emergence of a second area of high density (Fig. 11D). From these cumulative histograms, we conclude that in barrelless the highdensity domains are at the basis of a topographic representation of the whisker follicles.

\section{Discussion}

To elucidate the possible structural basis underlying the barrelless phenotype, we reconstructed TCAs in NOR and BRL mice. Comparing the population of handdrawn axons with the morphometrical data from the three-dimensional (3D)reconstructed axons confirms that the conclusions drawn from the quantitative analysis are representative for the total sample of labeled TCAs. These conclusions are the following: (1) The subcortical trajectory of BRL axons is the same as of NOR axons. TCAs in both strains have the same total axonal length, bear the same number of branches and axonal boutons. (2) BRL axons possess branches at the interface between white matter and layer VI, that were not observed in NOR. (3) The number of branches by which TCA axons in BRL reach layer IV is increased in comparison to NOR; also, the areal surface covered by these entering branches is larger in BRL than in NOR. (4) The comparison of the geometry between the two populations of TCA revealed that an action potential in BRL axons has to cross a smaller number of axonal bifurcations before reaching the terminal branches. (5) Within layer IV, BRL axons distribute axonal boutons over a larger cortical area, forming a terminal plexus in which the bouton density is lower when compared with the compact, high bouton dense terminal plexus invariably formed by NOR axons. However, NOR and BRL axons have the same high-density area of axonal boutons, and in both strains, axons with the cell bodies close together in the thalamus made their high-density areas in the same cortical region.

A number of the morphometric parameters (total length, number of branches, and axonal boutons) are indistinguishable between the two strains studied here and, interestingly, have an almost identical value for TCAs terminating in the primary visual cortex of the mouse (Antonini et al., 1999). TCA axons in the primary visual cortex of the tree shrew (Raczkowski and Fitzpatrick, 1990) and macaque (Blasdel and Lund, 1983) bear comparable numbers of boutons as the ax-
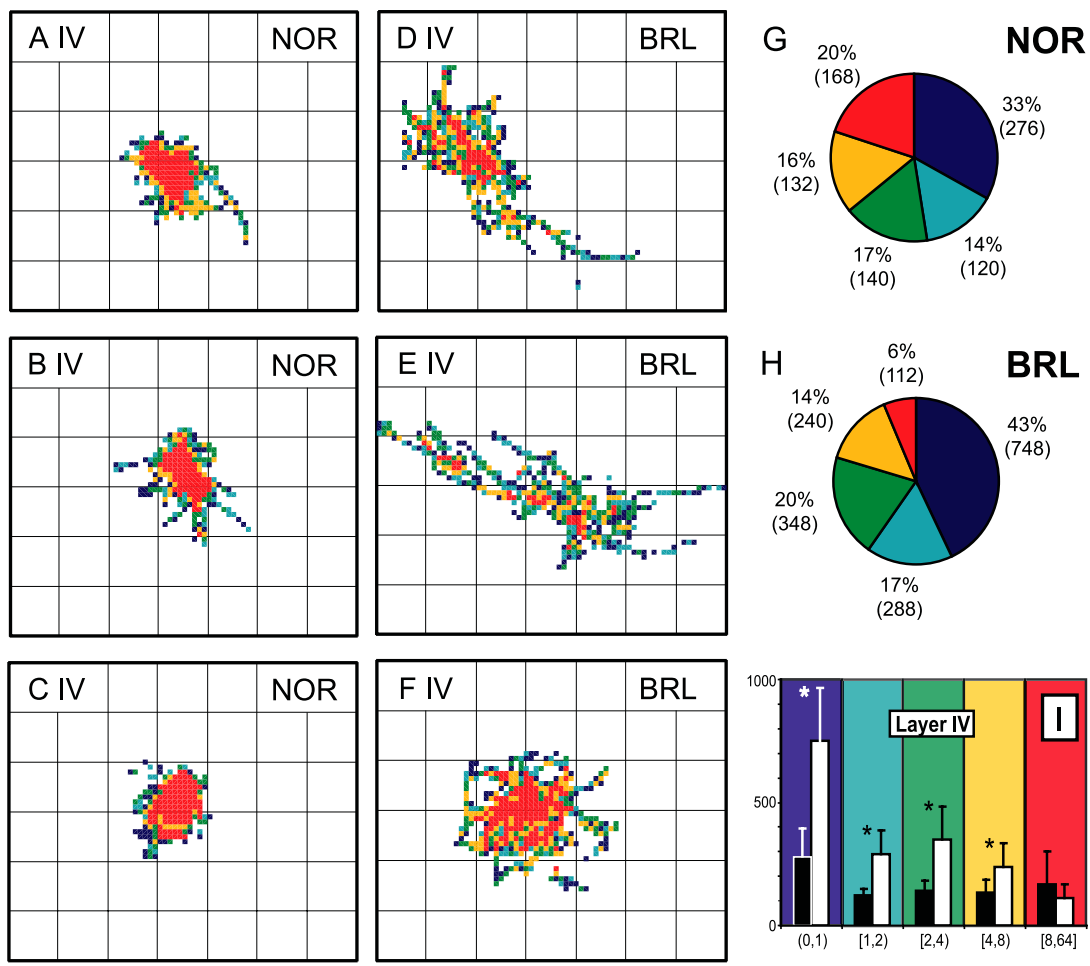

$A=(0,1]$

$B=(1,2]$

$C=(2,4]$

$D=(4,8]$

$E=(8,64]$
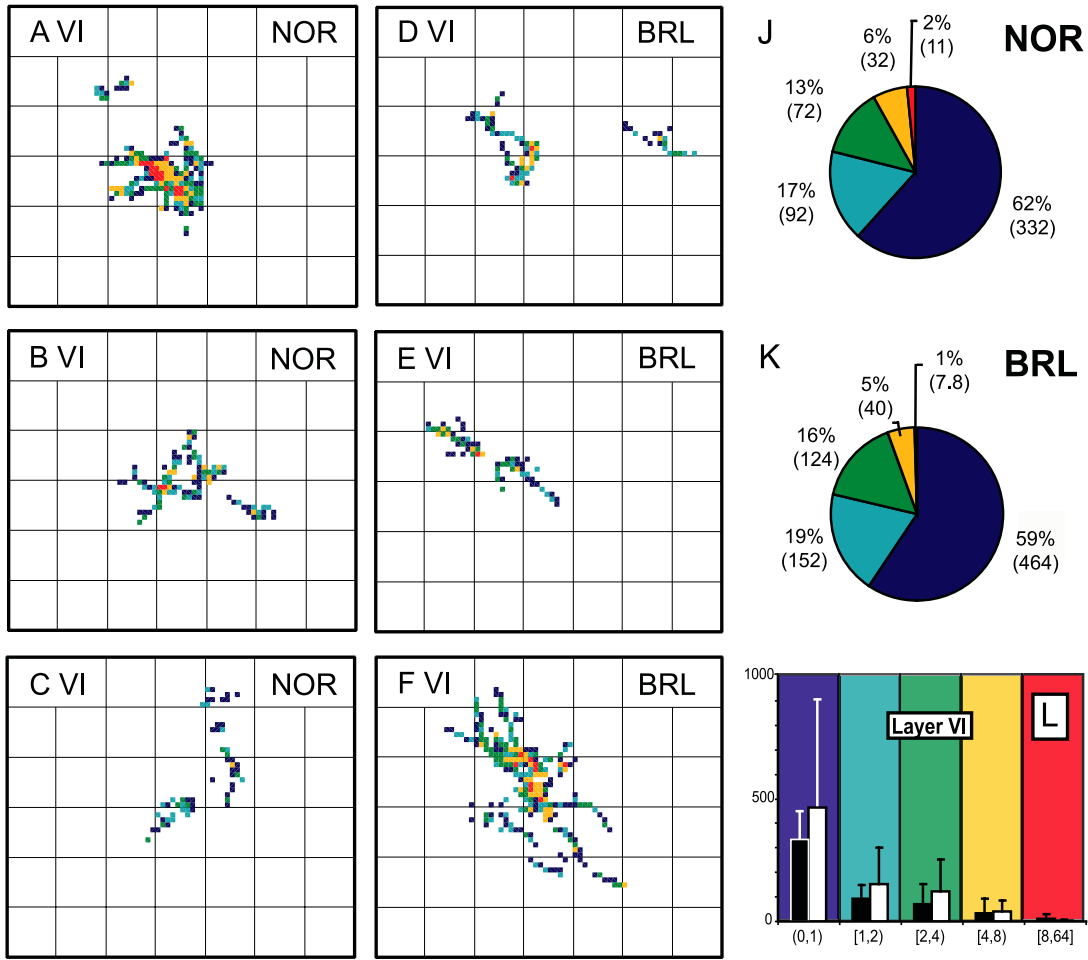

Figure 10. Two-dimensional density plots illustrating tangential distribution of the boutons of NOR and BRL axons within layer IV and VI. For individual axons, the density of boutons was determined separately for the plexus in layer IV and layer VI by calculating the number of boutons per $400 \mu \mathrm{m}^{2}$ area of tangential cortical surface. Distributions are displayed on a grid with a line distance of $200 \times 200 \mu \mathrm{m}$. The color bar gives the coding used for the five density ranges applied. AIV-CIV, Bouton distribution in layer IV of three NOR axons; DIV-FIV, distribution in layer IV of three BRL axons; AVI-CVI, distribution of boutons in layer VI of the NOR axons displayed above; DVI-FVI, distribution in layer VI of the three $B R L$ axons. The pie charts $(G, H, J, K)$ represent the mean percentages of each of the five density ranges as calculated for NOR and BRL plexuses separately. The mean absolute values of the surface areas in $100 \mu \mathrm{m}^{2}$ are given in parentheses. These latter values are displayed in the bar graphs $(I, L)$ allowing comparison between strains; the asterisks indicate the significant differences (one-way univariate analysis; $p<0.05$ ). Error bars indicate SD. 
A NOR : 2 axons

B BRL : 2 axons
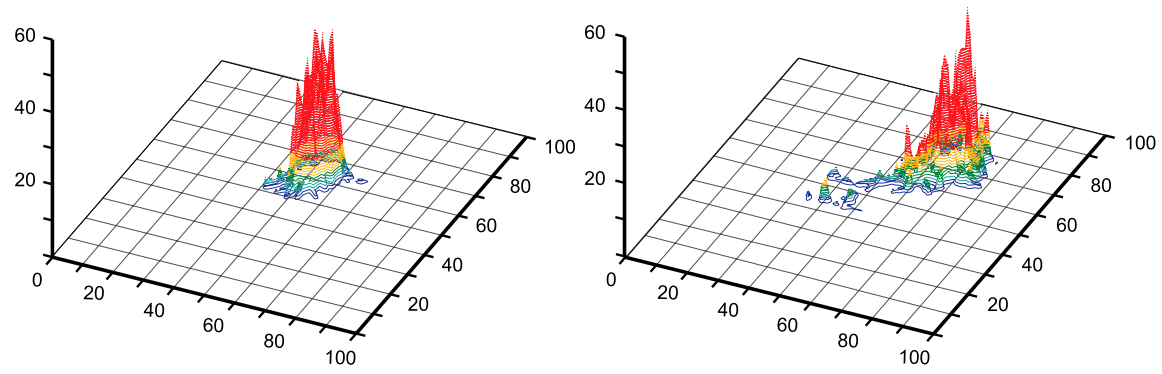

C BRL : 3 axons

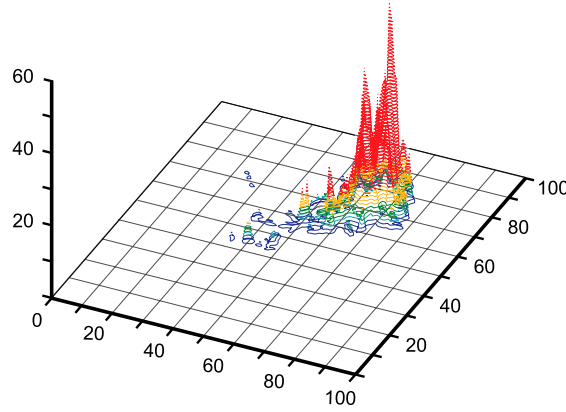

D BRL : 5 axons

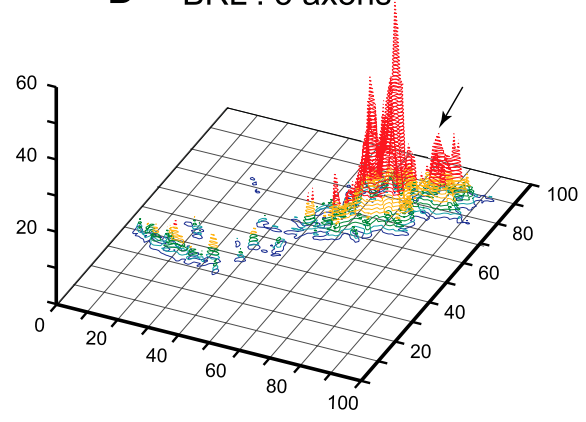

Figure 11. Three-dimensional density plots for NOR and BRL axons that reach the same region inside layer IV. $\boldsymbol{A}$, The representation of axonal plexus formed by two NOR axons in layer IV of the barrel cortex (NOR 53A and NOR53C). From this combined plexus, the bouton density was calculated per $400 \mu \mathrm{m}^{2}$ tangential cortical surface and expressed vertically. The color coding corresponds to the density ranges identified in Figure 10. The graph shows that both axons reached the same region inside layer IV where the sum of their boutons $(n=2701)$ reached a peak density of 39 boutons $/ 400 \mu \mathrm{m}^{2}$. The same procedure was applied for BRL axons: $\boldsymbol{B}, B R L 33$ and BRL 33D (number of boutons, 3238; maximal density, 44 boutons $/ 400 \mu \mathrm{m}^{2}$ ); $\boldsymbol{C}$, as $\boldsymbol{B}$ plus BRL 33A (number of boutons, 4410; maximal density, 57 boutons $/ 400 \mu \mathrm{m}^{2}$ ); $\boldsymbol{D}$, as ( plus BRL 33B and BRL 33E (number of boutons, 5919; maximal density, 60 boutons $/ 400 \mu \mathrm{m}^{2}$ ). This series of graphs shows the formation of a cortical area with a high bouton density in the BRL mouse that equals the size of the single barrel area delineated by the NOR axons in $\boldsymbol{A}$. The arrow in $\boldsymbol{D}$ points to the emergence of an additional barrel-like area attributable to the representation of axon BRL 33B and 33E. columnar shape as well as the topological relation between TCAs is established by progressive convergence inside the cortex. In the subcortical trajectory, TCAs with cell bodies close together in the thalamus may lose this spatial relationship. However, from the cortical entry point until their arrival at the boundary between layers IV and V, TCAs redirect to finally converge within the same barrel. This progressive convergence reflects the developmental sequence by which TCAs grow into the cortex and establish whiskerrelated patterns first in the deep layers before the arrival and pattern formation in layer IV (Agmon et al., 1993, 1995).

Several studies have identified the molecules involved in subcortical pathway finding of TCA (Dufour et al., 2003; Lopez-Bendito et al., 2006) and the signals that TCA follow inside the cortex to terminate in target layers IV and VI (Cang et al., 2005; Shimogori and Grove, 2005). It was further shown that TCA can correct an altered development both during their subcortical trajectory (Higashi et al., 2005) as well as their subsequent growth within cortex (for review, see Rash and Grove, 2006). Although our observations are made in adulthood, it seems that the BRL mutation has neither influenced the subcortical trajectory, nor the termination of TCAs in the appropriate cortical layers.

We showed that BRL mutation disrupts TCA morphology both below and inside layer IV. First, BRL TCAs possess supernumerary WM/VI branches that resemble ons studied here (see supplemental table, available at www. jneurosci.org as supplemental material). This transmodal and interspecies comparison indicates the constant nature of TCA axons in the vertebrate sensory pathways.

Although we analyzed the spatial distribution of axonal boutons in three dimensions, we included in Table 1 the mediolateral extent of our TCA allowing comparison with the literature. NOR axons have a mediolateral extent comparable with TCA in VI of the mouse (Antonini et al., 1999), the barrel cortex of the rat (Jensen and Killackey, 1987a), the primary visual cortex of the tree shrew (Raczkowski and Fitzpatrick, 1990), and the primary visual cortex of macaque (Blasdel and Lund, 1983). Combined with the comparison of the number of boutons, this indicates that the density of the projection of individual TCAs within a tangential area of primary sensory cortex is highly constant between cortical areas and between species. This conclusion is further strengthened by the linear correlation found between axonal length and tangential extent in both strains of mice studied here. Although variability exists between individual TCAs, their contribution to the innervation of the cortex clearly follows common rules. This interstrain/interspecies comparison predicts that variations in the impact of the thalamocortical projections in a given part of the primary sensory cortex are either attributable to a modification in the number of TCAs entering the cortex or the consequence of an alteration in the geometry of TCAs.

Two factors influence the final geometry of TCAs: sensory activity and intracortical neuronal transmission. In NOR, the those described for TCAs during development but that are normally not found in adults (Naegele et al., 1988; Senft and Woolsey, 1991a,b; Ghosh and Shatz, 1992; Catalano et al., 1996; Auladell et al., 2000). Some branches look similar to the "interstitial budding” branches described by O’Leary and Terashima, (1988). Second, the number of axonal branches by which BRL TCAs reach layer IV is increased, and together they form an area of entry in layer IV that is several times larger than that in NOR. Third, in layer IV, BRL axons extend across a cortical surface that is twice that in NOR. These observations indicate that developmental mechanisms as branch pruning/retraction (O'Leary and Terashima, 1988; Skaliora et al., 2000) may be deficient during infragranular and granular stages of TCA development in BRL. This is in agreement with the observations of Rebsam et al. (2002) and Lee et al. (2005) and congruent with the hypothesis that axonal pruning/retraction are mechanisms involved in geometrical shaping of TCA (Senft and Woolsey, 1991a,b; Skaliora et al., 2000; Portera-Cailliau et al., 2005).

These deficiencies can be explained by two, not mutually exclusive, mechanisms. It can be attributable to a dysfunction of BRL TCAs to respond to guidance molecules comparable with the maintenance of ectopic retinal projections in the superior colliculus of barrelless mice (Ravary et al., 2003). In this retinal projection, it was shown that BRL axons maintain aberrant branches because of a modified response to ephrin-5 signaling (Nicol et al. 2006). Alternatively, the extensive BRL arbor can be the consequence of synaptic dysfunction during the first postna- 
tal week when the induction of long-term potentiation was shown to be impaired in the BRL mutant (Lu et al., 2003), a hypothesis that further underlines the role of activity-dependent synaptic plasticity in shaping TCAs (Erzurumlu and Kind, 2001).

In layer IV, a TCA in NOR creates a compact terminal plexus with the size of a barrel, $20 \%$ of which is characterized by an area of high density of thalamocortical (TC) boutons. In BRL, the layer IV plexus has an area comparable with up to 2.5 barrels and only $6 \%$ of this area is characterized by a high density of TC boutons. The high degree of divergence of TCA termination in layer IV is the basis for the lack of segregation of whisker input to individual layer IV neurons in BRL strain (Welker et al., 1996). The total areal extent of the high-density domains in BRL and NOR are similar, indicating that this aspect of the TCA plexus tends to be resistant to the genetic variation. A similar result has been described by Antonini and Stryker (1993), who demonstrated that decreasing sensory activation in the visual pathway did not alter the high-density area of geniculocortical axons either.

However, the current study reveals that, in BRL, TCAs of neighboring thalamic neurons form a compact area of highdensity innervation that has the tangential size of a single barrel. We propose that this high-density area underlies the topologic parcellation of SI in barrelless mice shown previously (Welker et al., 1996). This implies that not all parts of the layer IV plexus of an individual TCA contribute in equal manner to the establishment of a functional map in SI. This notion is in harmony with the Gaussian distribution of TCA boutons in SI of the monkey cortex (Garraghty and Sur, 1990), where the peak of the curve coincides with the corresponding part of the sensory periphery. Enlargement of the functional representation during adult plasticity may therefore be the consequence of a proportional increase in the activation of the part of the TCA termination that has a low bouton density (Garraghty et al., 1989; Garraghty and Sur, 1990; Rausell and Jones, 1995; Jones and Pons, 1998).

In conclusion, we propose that developmental mechanisms that shape thalamocortical axons before their arrival in cortical layer IV are deficient in the BRL mouse, but that thalamic neighborhood relationships are maintained in the cortical projection, in which the spatial distribution of TC boutons underlie the establishment of a functional map of the mystacial whisker follicles.

\section{References}

Abdel-Majid RM, Leong WL, Schalkwyk LC, Smallman DS, Wong ST, Storm DR, Fine A, Dobson MJ, Guernsey DL, Neumann PE (1998) Loss of adenylyl cyclase I activity disrupts patterning of mouse somatosensory cortex. Nat Genet 19:289-291.

Adams NC, Lozsadi DA, Guillery RW (1997) Complexities in the thalamocortical and corticothalamic pathways. Eur J Neurosci 9:204-209.

Agmon A, Yang LT, O’Dowd DK, Jones EG (1993) Organized growth of thalamocortical axons from the deep tier of terminations into layer IV of developing mouse barrel cortex. J Neurosci 13:5365-5382.

Agmon A, Yang LT, Jones EG, O’Dowd DK (1995) Topological precision in the thalamic projection to neonatal mouse barrel cortex. J Neurosci 15:549-561.

Antonini A, Stryker MP (1993) Development of individual geniculocortical arbors in cat striate cortex and effects of binocular impulse blockade. J Neurosci 13:3549-3573.

Antonini A, Gillespie DC, Crair MC, Stryker MP (1998) Morphology of single geniculocortical afferents and functional recovery of the visual cortex after reverse monocular deprivation in the kitten. J Neurosci 18:9896-9909.

Antonini A, Fagiolini M, Stryker MP (1999) Anatomical correlates of functional plasticity in mouse visual cortex. J Neurosci 19:4388-4406.

Auladell C, Perez-Sust P, Super H, Soriano E (2000) The early development of thalamocortical and corticothalamic projections in the mouse. Anat Embryol (Berl) 201:169-179.

Blasdel GG, Lund JS (1983) Termination of afferent axons in macaque striate cortex. J Neurosci 3:1389-1413.

Cang J, Renteria RC, Kaneko M, Liu X, Copenhagen DR, Stryker MP (2005) Development of precise maps in visual cortex requires patterned spontaneous activity in the retina. Neuron 48:797-809.

Cases O, Vitalis T, Seif I, De Maeyer E, Sotelo C, Gaspar P (1996) Lack of barrels in the somatosensory cortex of monoamine oxidase A-deficient mice: role of a serotonin excess during the critical period. Neuron 16:297-307.

Catalano SM, Robertson RT, Killackey HP (1996) Individual axon morphology and thalamocortical topography in developing rat somatosensory cortex. J Comp Neurol 367:36-53.

Caviness Jr VS (1975) Architectonic map of neocortex of the normal mouse. J Comp Neurol 164:247-263.

Caviness Jr VS, Frost DO (1980) Tangential organization of thalamic projections to the neocortex in the mouse. J Comp Neurol 194:335-367.

Dolleman-Van der Weel MJ, Wouterlood FG, Witter MP (1994) Multiple anterograde tracing, combining Phaseolus vulgaris leucoagglutinin with rhodamine- and biotin-conjugated dextran amine. J Neurosci Methods 51:9-21.

Dufour A, Seibt J, Passante L, Depaepe V, Ciossek T, Frisen J, Kullander K, Flanagan JG, Polleux F, Vanderhaeghen P (2003) Area specificity and topography of thalamocortical projections are controlled by ephrin/Eph genes. Neuron 39:453-465.

Erzurumlu RS, Kind PC (2001) Neural activity: sculptor of "barrels" in the neocortex. Trends Neurosci 24:589-595.

Garraghty PE, Sur M (1990) Morphology of single intracellularly stained axons terminating in area $3 \mathrm{~b}$ of macaque monkeys. J Comp Neurol 294:583-593.

Garraghty PE, Pons TP, Sur M, Kaas JH (1989) The arbors of axons terminating in middle cortical layers of somatosensory area $3 \mathrm{~b}$ in owl monkeys. Somatosens Mot Res 6:401-411.

Ghosh A, Shatz CJ (1992) Pathfinding and target selection by developing geniculocortical axons. J Neurosci 12:39-55.

Hannan AJ, Blakemore C, Katsnelson A, Vitalis T, Huber KM, Bear M, Roder J, Kim D, Shin HS, Kind PC (2001) PLC-beta 1, activated via mGluRs, mediates activity-dependent differentiation in cerebral cortex. Nat Neurosci 4:282-288.

Higashi S, Hioki K, Kurotani T, Kasim N, Molnar Z (2005) Functional thalamocortical synapse reorganization from subplate to layer IV during postnatal development in the reeler-like mutant rat (shaking rat Kawasaki). J Neurosci 25:1395-1406.

Humphrey AL, Sur M, Uhlrich DJ, Sherman SM (1985) Projection patterns of individual $\mathrm{X}$ - and Y-cell axons from the lateral geniculate nucleus to cortical area 17 in the cat. J Comp Neurol 233:159-189.

Iwasato T, Datwani A, Wolf AM, Nishiyama H, Taguchi Y, Tonegawa S, Knopfel T, Erzurumlu RS, Itohara S (2000) Cortex-restricted disruption of NMDAR1 impairs neuronal patterns in the barrel cortex. Nature 406:726-731.

Jensen KF, Killackey HP (1987a) Terminal arbors of axons projecting to the somatosensory cortex of the adult rat. I. The normal morphology of specific thalamocortical afferents. J Neurosci 7:3529-3543.

Jensen KF, Killackey HP (1987b) Terminal arbors of axons projecting to the somatosensory cortex of the adult rat. II. The altered morphology of thalamocortical afferents following neonatal infraorbital nerve cut. J Neurosci 7:3544-3553.

Jones EG (1985) Principle of thalamic organization. In: The thalamus, pp 100-113. New York: Plenum.

Jones EG (1986) Connectivity of the primate sensory-motor cortex. In: Cerebral cortex, Vol 5, Sensory-motor areas and aspects of cortical connectivity (Jones EG, Peters Alan, eds), pp 127-147. New York: Plenum.

Jones EG, Pons TP (1998) Thalamic and brainstem contributions to largescale plasticity of primate somatosensory cortex. Science 282:1121-1125.

Lee LJ, Iwasato T, Itohara S, Erzurumlu RS (2005) Exuberant thalamocortical axon arborization in cortex-specific NMDAR1 knockout mice. J Comp Neurol 485:280-292.

Lopez-Bendito G, Cautinat A, Sanchez JA, Bielle F, Flames N, Garratt AN, Talmage DA, Role LW, Charnay P, Marin O, Garel S (2006) Tangential neuronal migration controls axon guidance: a role for neuregulin-1 in thalamocortical axon navigation. Cell 125:127-142. 
Lu HC, She WC, Plas DT, Neumann PE, Janz R, Crair MC (2003) Adenylyl cyclase I regulates AMPA receptor trafficking during mouse cortical "barrel" map development. Nat Neurosci 6:939-947.

Maier DL, Mani S, Donovan SL, Soppet D, Tessarollo L, McCasland JS, Meiri KF (1999) Disrupted cortical map and absence of cortical barrels in growth-associated protein (GAP)-43 knockout mice. Proc Natl Acad Sci USA 96:9397-9402.

McIlvain VA, Robertson DR, Maimone MM, McCasland JS (2003) Abnormal thalamocortical pathfinding and terminal arbors lead to enlarged barrels in neonatal GAP-43 heterozygous mice. J Comp Neurol 462:252-264.

Naegele JR, Jhaveri S, Schneider GE (1988) Sharpening of topographical projections and maturation of geniculocortical axon arbors in the hamster. J Comp Neurol 277:593-607.

Nicol X, Muzerelle A, Rio JP, Metin C, Gaspar P (2006) Requirement of adenylate cyclase 1 for the ephrin-A5-dependent retraction of exuberant retinal axons. J Neurosci 26:862-872.

O'Leary DD, Terashima T (1988) Cortical axons branch to multiple subcortical targets by interstitial axon budding: implications for target recognition and "waiting periods." Neuron 1:901-910.

Pinault D (1996) A novel single-cell staining procedure performed in vivo under electrophysiological control: morpho-functional features of juxtacellularly labeled thalamic cells and other central neurons with biocytin or Neurobiotin. J Neurosci Methods 65:113-136.

Portera-Cailliau C, Weimer RM, De Paola V, Caroni P, Svoboda K (2005) Diverse modes of axon elaboration in the developing neocortex. PLoS Biol 3:e272.

Raczkowski D, Fitzpatrick D (1990) Terminal arbors of individual, physiologically identified geniculocortical axons in the tree shrew's striate cortex. J Comp Neurol 302:500-514.

Rash BG, Grove EA (2006) Area and layer patterning in the developing cerebral cortex. Curr Opin Neurobiol 16:25-34.

Rausell E, Jones EG (1995) Extent of intracortical arborization of thalamocortical axons as a determinant of representational plasticity in monkey somatic sensory cortex. J Neurosci 15:4270-4288.

Ravary A, Muzerelle A, Herve D, Pascoli V, Ba-Charvet KN, Girault JA,
Welker E, Gaspar P (2003) Adenylate cyclase 1 as a key actor in the refinement of retinal projection maps. J Neurosci 23:2228-2238.

Rebsam A, Seif I, Gaspar P (2002) Refinement of thalamocortical arbors and emergence of barrel domains in the primary somatosensory cortex: a study of normal and monoamine oxidase a knock-out mice. J Neurosci 22:8541-8552.

Senft SL, Woolsey TA (1991a) Growth of thalamic afferents into mouse barrel cortex. Cereb Cortex 1:308-335.

Senft SL, Woolsey TA (1991b) Computer-aided analyses of thalamocortical afferent ingrowth. Cereb Cortex 1:336-347.

Shimogori T, Grove EA (2005) Fibroblast growth factor 8 regulates neocortical guidance of area-specific thalamic innervation. J Neurosci 25:6550-6560.

Skaliora I, Adams R, Blakemore C (2000) Morphology and growth patterns of developing thalamocortical axons. J Neurosci 20:3650-3662.

Tettoni L, Lehmann P, Houzel JC, Innocenti GM (1996) Maxsim, software for the analysis of multiple axonal arbors and their simulated activation. J Neurosci Methods 67:1-9.

Van der Loos H, Dorfl J, Welker E (1984) Variation in pattern of mystacial vibrissae in mice. A quantitative study of ICR stock and several inbred strains. J Hered 75:326-336.

Welker E, Armstrong-James M, Bronchti G, Ourednik W, GheorghitaBaechler F, Dubois R, Guernsey DL, Van der Loos H, Neumann PE (1996) Altered sensory processing in the somatosensory cortex of the mouse mutant barrelless. Science 271:1864-1867.

White EL, Weinfeld E, Lev DL (2004) Quantitative analysis of synaptic distribution along thalamocortical axons in adult mouse barrels. J Comp Neurol 479:56-69.

Woolsey TA, Van der Loos H (1970) The structural organization of layer IV in the somatosensory region (SI) of mouse cerebral cortex. The description of a cortical field composed of discrete cytoarchitectonic units. Brain Res 17:205-242.

Yang Z, Seif I, Armstrong-James M (2001) Differences in somatosensory processing in S1 barrel cortex between normal and monoamine oxidase $\mathrm{A}$ knockout (Tg8) adult mice. Cereb Cortex 11:26-36. 